\title{
A compact thermal lithium-beam source using a solid A/Li alloy for Li sublimation
}

\author{
R.P. Schorn, E. Hintz, S. Musso, and B. Schweer \\ Institut für Plasmaphysik der Kernforschungsanlage Jülich GmbH, Association EURATOM-XFA, Postfach \\ 1913, D.5170 Julich, Federal Republic of Gemany
}

(Received 13 April 1989; accepted for publication 22 June 1989)

\begin{abstract}
Thermal lithium beams are widely used in physics for different purposes. To overcome certain disađ̃vantages of conventional liquid-lithium ovens concerning handing, conditioning, and lifetime of the Li flling, a solid Li-evaporation device was constructed. An aluminum-based alloy, which contains about 9 at. \% of lithium, is employed. The oven can easily be mounted in any position, and Liatoms can be injected in any desired direction. The parameters of the beam (axial and radial fux profiles) have been measured as a function of temperature and time. At a distance of $100 \mathrm{~mm}$ from the oven, Lifux densities of more than $2 \times 10^{14} \mathrm{~cm}^{-2} \mathrm{~s}^{-1}$ have been achieved, lasting for more than 16 days of continued operation. The full beam divergence at half maximum is $8^{\circ}$. As a typical appication of the device in plasma diagnostics, radial electron density pronles have been measured in the scrape-off layer of the TEXTOR tokamak.
\end{abstract}

\section{INTRODUCTION}

Thermal lithium beams of the conventional type are empioyed in many experiments by evaporating pure liquid lithium. Such an oven of the "classic" style has several disadvantages. Liquid lithium is a chemically aggressive substance, especially at elevated temperatures. Cleaning and refilling of the Li reservoir is time consuming and dificult. Elemental lithium being exposed to air is always covered by impurities such as oxides, hydroxides, and compounds of lithium and nitrogen. The first heating of the oven will not oniy lead to evaporation of lithium, but also to that of the impurities. Furthermore, complications arise, when the beam is to be injected downwards into an experiment. Then, the li vapor has to be guided by curved ducts, which have to be at a higher temperature than the oven itself. This may lead to a complex experimental setup.

These difficulties can be overcome by applying a solid $\mathrm{Al} / \mathrm{Li}$ alloy. When the Li concentration is less than $10 \mathrm{at} . \%$, this material remains in the solid state for temperatures below $620^{\circ} \mathrm{C}$, while the lithium component is sublimated. Based on this behavior, a compact thermal lithium-beam source using $\mathrm{Al} / \mathrm{Li}$ has been constructed. Its design and beam properties, as well as some basic results obtained with it in plasma diagnostics, shall be presented in the following sections.

\section{CHARACTERISTICS OF THE LITHUUM ATOMIC BEAM OVEN}

As already pointed out in a previous publication, 'the volatile lithium component of an aluminum-based soid alloy, containing a small amount of lithium, starts to evaporate, when the temperature is raised above $300^{\circ} \mathrm{C}$. According to the phase diagram of the $\mathrm{Al} / \mathrm{Li}$ system ${ }^{2}$ (see Fig. 1), a liquid phase does not exist for temperatures below $620^{\circ} \mathrm{C}$ and lithium concentrations below 10 at. \%. In this regime the alloy forms an $\alpha$ phase at elevated temperatures based on the solid-aluminum foc structure, and a mixed phase consisting of the $\alpha$ phase and the intermetallic compound AlLi the $\beta$ phase), which is based on the NaTl-type structure. In both phases, the diffusion coefficient of $\mathrm{Li}$ in $\mathrm{Al}$ is high enough $\left(5 \times 10^{-9} \mathrm{~cm}^{2} / \mathrm{s}\right.$ at $\left.540^{\circ} \mathrm{C}^{3,4}\right)$ to compensate evaporation losses by bulk diffusion. According to Ref. 4 , the value is nearly independent of the Li concentration. These properties open a possibility to create a thermal lithium atom beam without using the hard-to-handle and chemically aggressive liquid lithium.

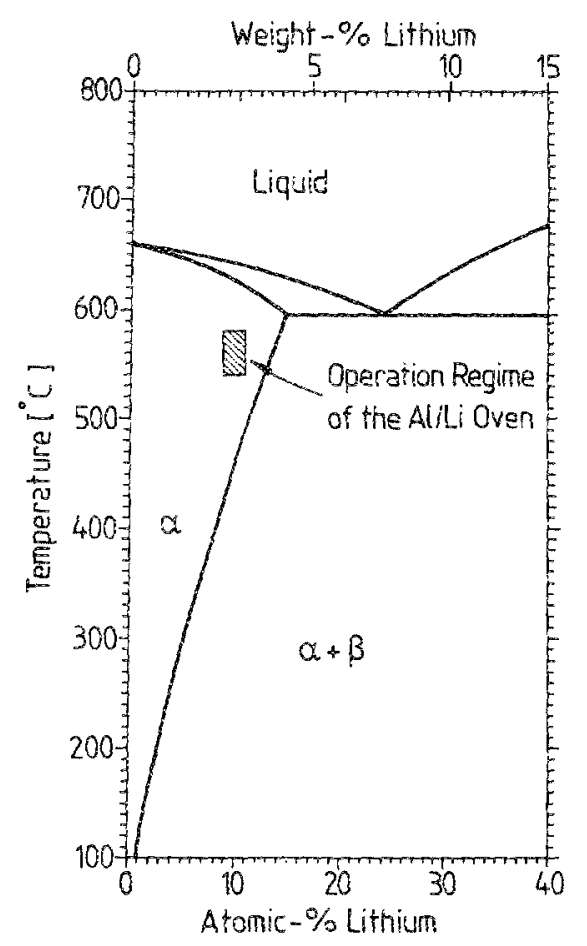

FIG. 1. Part of the phase diagram of the Al/ hi system as a redraw from Ref. 2. The $\mathrm{Al} / \mathrm{Li}$ material is operated in the solid $\alpha$ phase at temperatures close below $600^{\circ} \mathrm{C}$. 
In Ref. I we have presented flux densities of evaporated monatomic neutral lithium measured by means of laser-induced fluorescence spectroscopy (LIF), calibrated by Rayleigh scattering in argon gas. As another independent absolute calibration method, covering also evaporated ions and $\mathrm{Li}_{2}$, a quartz oscillator microbalance was used. The principle of the method is described, e.g., in Ref. 5 . In order to compare results with those obtained in previous measurements ', a similar evaporation geometry was used: A plane $\mathrm{Al} / \mathrm{Li}$ disk ( $90 \mathrm{~mm}$ in diameter, $2 \mathrm{~mm}$ thickness) was heated clectrically. The surface temperature was monitored by a thermocouple and kept at constant level by an automatic heating system. The quartz plate (of circular shape and $6 \mathrm{~mm}$ in diameter) was mounted at a distance of $z=100 \mathrm{~mm}$ from the emitting disk on the center axis ( $z$ axis). The frequency detuning of the quartz oscillator was used to calculate the lithium flux density hitting the quartz surface.

The quartz plate is exposed to lithium evaporation from an extended area. The concept of a point source is not valid here. In order to derive the evaporation rate $\Phi(0)$ at the disk surface from the measured value $\Phi(z)$ at the distance $z$, the $\mathrm{Li}$ emission has to be integrated over the evaporating surface. Provided that the angular emission profile of each surface element is proportional to the cosine of the emission angle, one obtains, for the fiux density along the center axis, ${ }^{6}$

$$
\Phi(z)=\Phi(0)\left[1-z^{2} /\left(R^{2}+z^{2}\right)\right]
$$

where $R$ is the radius of the disk, and $z$ is the distance from the surface to the position of flux measurement on the axis. In our case, fiux densities at $z=100 \mathrm{~mm}$ have to be multiplied by a factor of 5.9 to get the surface values. Figure 2 shows lithium evaporation rates obtained by this way for temperatures between 400 and $580^{\circ} \mathrm{C}$ in an Arrhenius plot. LIF results from Ref. 1 are also shown.

The two curves differ by about $40 \%$, where the LIF technique yields the lower values. To explain this, several reasons have to be considered: The major part of the absolute error is due to the Rayleigh scattering calibration method of LIF. Uncertainties in determining the laser power density (i.e. laser energy, cross section of the beam, and pulse duration) as well as statistical errors of the scattered radiation add up to an error bar of the Li fux of about $\pm 40 \%$. The error associated with the quartz balance technique (i.e., determinaton of resonance frequency decay, accuracy of the quartz' material constants) can be estimated to be less than $\pm 10 \%$. As Li surfaces easily react with oxygen, nitrogen, and water vapor being contained in the residual gas (here $10^{-7}$ mbar total pressure), fluxes recorded with a quarce balance may also include certain amounts of adsorbed gases. In our case, however, it can be shown that this amount of "additional flux" is less than $10^{14} \mathrm{~cm}^{-2} \mathrm{~s}$ " and can be neglected. The content of $\mathrm{Li}^{+}$and $\mathrm{Li}_{2}$ in the beam could not be monitored, but is expected to be less than 10\%. Both techniques yield absolute flux values, which agree within the limits of the error bars (see Fig. 2). Moreover, they both reveal about $1.5 \mathrm{eV}$ as energy of vaporization, being typical for elemental lithium. As already concluded in Ref. 1, the alloy surface is predominantly covered by a complete lithium

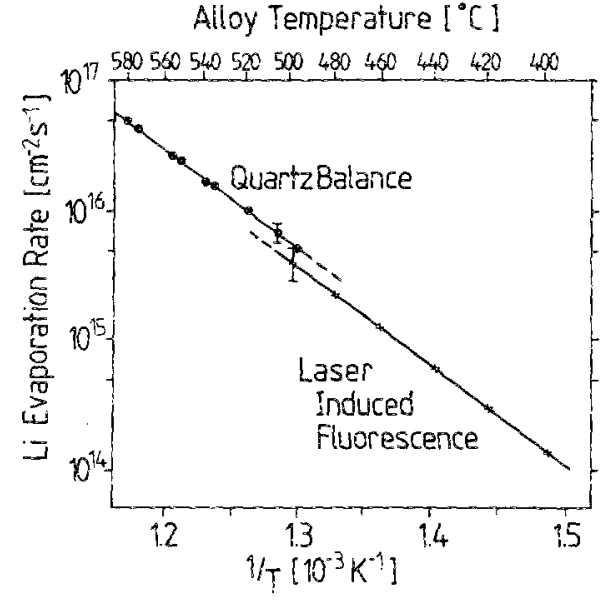

Fig. 2. Temperature-dependent evaporation rates of lithium emitted out of a solid Al/Li alloy. Li fluxes have been measured with laser-induced fuorescence spectroscopy and a quartz microbalance.

overlayer as a result of combined segregation and diffusion processes.

The experimentally determined $\mathrm{Li}$ evaporation rates presented above encouraged us to use a closed cavity oven geometry. Lithium then sublimates from an inner surface, and in contrast to the plane disk emitting a broad (cosine) beam into vacuum, a collimation can be achieved. In this way, also the lifetime of the alloy material should be increased. The oven is of a compact cylindrical shape. It is made of a stainless-steel housing heated by a thermocoax and an exchangeable $\mathrm{Al} / \mathrm{Li}$ alloy insert containing about 9 at. \% of lithium. The material, which is applied in aerospace industry, is available from several aluminum producing companies. We have used $\mathrm{Al} / \mathrm{Li}$ manufactured by Ref. 7 . The temperature is measured close to the inner alloy surface by means of a thermocouple. Lithium effuses through a cylindrical collimation duct, which in our case has a diameter of $d=5 \mathrm{~mm}$ and a length of $l=15 \mathrm{~mm}$. A schematic setup of the oven device is shown in Fig. 3.

Again, $L i$ flux densities $\Phi$ were measured with a quartz oscillator microbalance at a distance of $z=100 \mathrm{~mm}$. The first point of interest was the temporal behavior of $\Phi$. We continuously operated the oven with an alloy mass containing $1.2 \mathrm{~g}$ of lithium for more than 16 days at a constant temperature of $540^{\circ} \mathrm{C}$. Figure 4 shows the result. After a steep decrease during the first 2 days, a slowly decreasing flux value of around $1 \times 10^{14}-1.5 \times 10^{14} \mathrm{~cm}^{-2} \mathrm{~s}^{-1}$ is reached. Elevated flux values at the start of operation may be attributed to evaporation of impurities of higher atomic mass, such as lithium oxides, lithium hydroxides, and compounds of lithium and nitrogen, which initially are present on the alloy surface before the first heating. The almost exponential decay of recorded fluxes during the first few days gives rise to this assumption. Using the full spatial beam divergence of $\theta=8^{\circ}$ at half maximum (see below), the total evaporated lithium mass can be calculated by integrating the curve in Fig. 4 with respect to time and area. In our case $3 \times 10^{20}$ atoms, which is less than $0.5 \%$ of the alloy insert's total lithium content, have been emitted through the effusion duct during the 16 days of operation. Thus, we are en- 


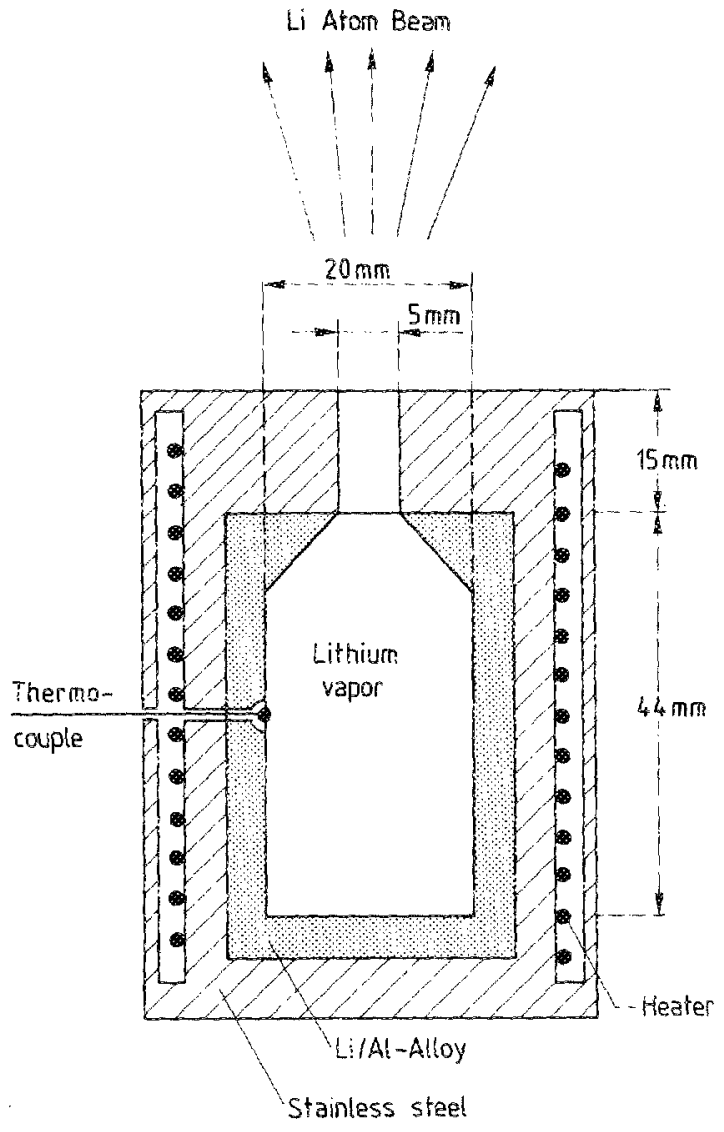

Fig. 3. Setup of the Al/Li oven: An exchangeable alloy insert is heated electrically with a thernocoax. The temperature is measured close to the inner insert surface. The efusion duct used here was $5 \mathrm{~mm}$ in diameter and $15 \mathrm{~mm}$ in length.

couraged to estimate the effective operation period (i.e. the lifetime) of one insert to be of the order of several months. Interrupting the evaporation process by lowering the temperature, e.g., at weekends, have no effect on the evaporation rate after restarting.

Next, we have investigated the temperature dependence of the lithium emission by the solid oven using the quartz

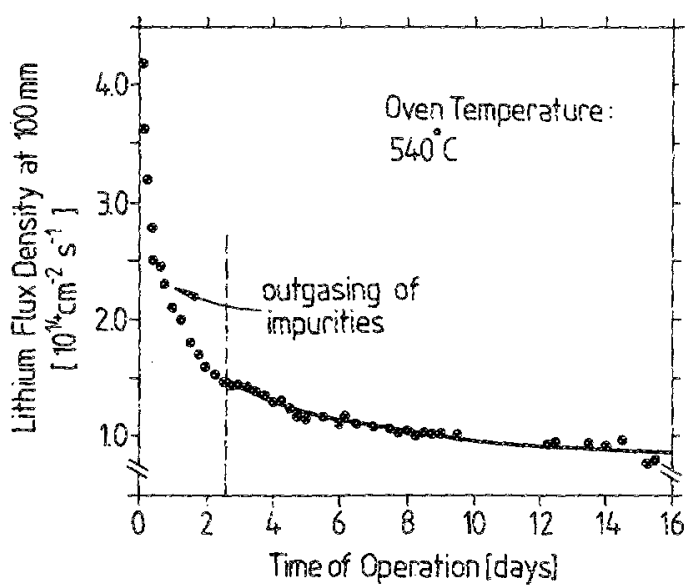

Fic. 4. Longtime performance of the lithium sublimation at $540^{\circ} \mathrm{C}$. The steep decrease during the first 2 days is attributed to evaporation of impurities such as oxides and other compounds of lithium.

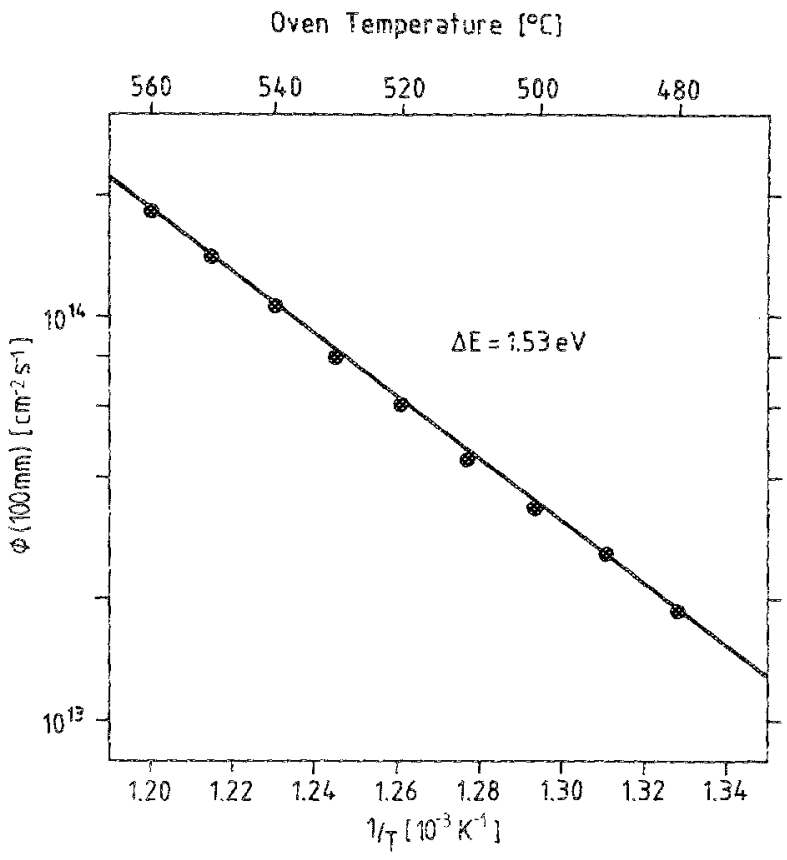

Fig. 5. Femperature dependence of the lithium emission of the oven. The energy of vaporization is deterinined to be $1.53 \mathrm{eV}$. Fluxes have been measured with a quartz microbalance at $z==100 \mathrm{~mm}$.

balance. Figure 5 shows Li fiux densities in the temperature range of $480-560^{\circ} \mathrm{C}$ measured at a normal distance of 100 $\mathrm{mm}$. As expected, they follow the well-known Arrhenius relation

$$
\Phi\left(7^{\prime}\right)=\Phi_{0} \exp \left(-\Delta E^{*} / k T\right) .
$$

where $T$ is the absolute temperature of the inner alloy surface. We derive an energy of vaporization of $\Delta E=1.53 \mathrm{eV}$, which is in good agreement with the standard value for elemental lithium $\left(1.54 \mathrm{eV}^{8}\right)$. The lithium layer deposited on the quartz balance's surface was analyzed by means of PIXE (proton induced $x$-ray emission), in order to ensure that the beam did not contain other elements beyond an acceptable percentage. These measurements were kindly performed by the Max-Planck-Institut für Plasmaphysik in Garching. ${ }^{9}$ After the above mentioned "conditioning time" of roughly 2 days, when probably also impurities are evaporated, we found that the aluminum particle content in the beam was less than $10^{-6}$ and the atomic concentration of other impurities $\left(Z n, A r, S\right.$, and $M g$ ) was less than $10^{-4}$ in total. A coverage of the quartz piate by oxygen, hydrogen, and nitrogen could not be monitored with PIXE. The quartz' surface analysis with PLXE and the outgassing behavior of the $\mathrm{Al} / \mathrm{Li}$ material lead us to the conclusion that the thermal beam predominantly consists of lithium after the above-mentioned conditioning time.

In Fig. 6 the spatial decay of $\Phi(z)$ along the center axis is depicied. Flux density values are normalized to the $z=100 \mathrm{~mm}$ position. The knowledge of this curve is important for application of the beam in plasma diagnositcs, e.g., when absolute electron densities have to be calculated from recorded lithium photon emission profiles (for details see Sec. II). In the examined distance of $40-140 \mathrm{~mm}$ from the effusion duct, fluxes vary by a factor of roughly 7 . To obtain 


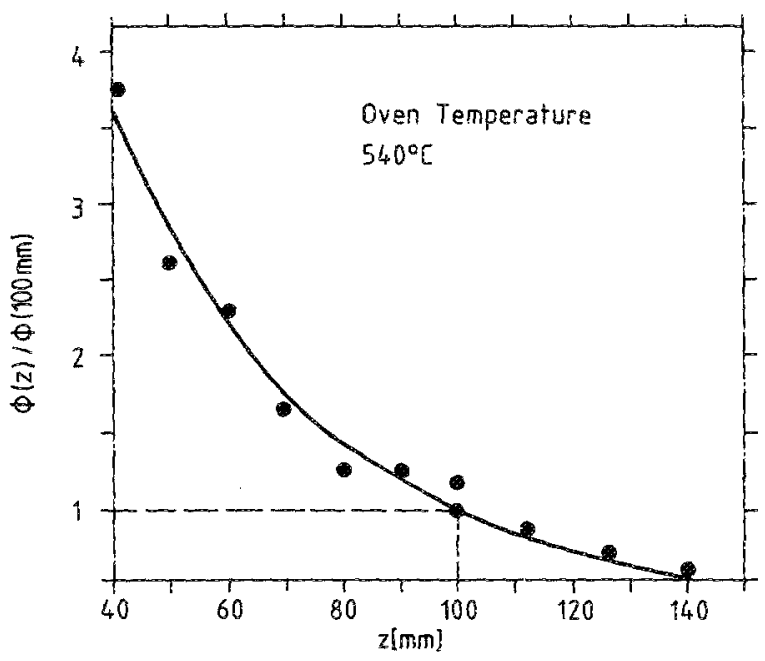

FIG. 6. Spatial decay of the lithium fux density along the center axis

the solid curve in Fig. 6, Eq. (1) was generalized using a $\cos ^{n}$-emission profile and fitted to the data points. This profile has been obtained again with the quartz balance. The oven was mounted on a manipulator, which could be moved in the $z$ direction.

The radial dimensions of the lithium beam have also to be known. As the quartz balance does not give sufficient spatial resolution (the quartz disk has a diameter of $6 \mathrm{~mm}$ ), and the water-cooled supporting system of the oscillator could not be moved radially, a so-called "Langmuir-Taylor detector" was built. Due to their relatively low energy of ionization, lithium atoms are easily ionized when hitting a hot platinum surface: High efficiencies are obtained when a metal is used, the work function of which approaches the energy of ionization of lithium. This is described quantitatively by the Saha-Langmuir equation. ${ }^{10}$ We used a platinum wire of $0.5-\mathrm{mm}$ diameter, which was heated electrically to temperatures around $1800 \mathrm{~K}$. The active length being hit by lithium atoms was limited to $1 \mathrm{~mm}$. Ionized $\mathrm{Li}$ atoms were extracted by a catcher electrode at $500 \mathrm{~V}$. The measured current is proportional to the lithium flux impinging onto the platinum wire. The Langmuir-Taylor detector was mounted on a radial manipulator system, so that beam profiles could be recorded also in this direction. Figure 7 shows three radial profiles at distances of $z=50,100$, and $150 \mathrm{~mm}$, respectively. At $150 \mathrm{~mm}$, which is the outmosi distance to be used at the TEXTOR tokamak (see below), the lithium beam has a diameter of $18 \mathrm{~mm}$ FWHM. A beam divergence of $8^{\circ}$ FWHM can be calculated from the data in Fig. 7. Beam divergences and radial profiles can be altered by changing the dimensions of the effusion duct. A detailed description of the measurements using a Langmuir-Taylor detector can be found in Ref. 11.

It should be noted that the measurements of radial and axial fiux profiles are not totally independent of each other. The sensitive quartz surface being exposed to the $\mathrm{Li}$ beam has a finite diameter of $6 \mathrm{~mm}$, while the platinum wire of the Langmuir-Taylor detector extends to $1 \mathrm{~mm}$. Expecially at closer distances from the oven's exit duct, integration over the spatial emission profile leads to altered radial and axial profiles as a consequence.

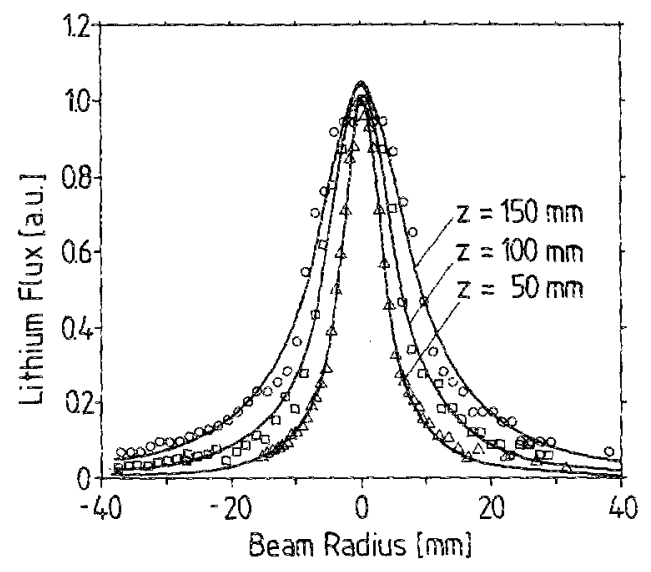

FIG. 7. Radial profiles of the lithium beam at three different distances from the effusion duct. The profles are normalized to unity. The beam's full divergence at half maximum is calculated to be $8^{\circ}$. Fluxes have been measured with a Langmuir-Taylor detector.

\section{APPLICATION TO THE TEXTOR TOKAMAK: MEASURING ELECTRON DENSITY PROFILES}

The Al/Li oven described above can be used successfully in plasma diagnostics. Here, one application is the measurement of electron density profiles in the boundary region of tokamaks: The performance of present day nuclear fusion experiments of the tokamak type suffers from plasma-wall contact, leading to plasma contamination by wall atoms. When studying these interactions, the knowledge of $n_{e}$ profiles is essential for a description of the boundary layer. Diagnostic methods to determine $n_{e}$ with a high spatial resolution have been developed in the past, using lithium atom beams of different energies (thermal, laser ablated suprathermal, and several $\mathrm{keV}$ ). They are injected radially into the scrape-off layer plasma. The principle of the method ${ }^{12-16}$ and its application to TEXTOR have been described in detail previously. ${ }^{13-45,17,18}$ Therefore, only a short outline shall be given: Due to collisions with plasma electrons, a certain fraction of the $\mathrm{Li}$ atoms, which initially are in the $2{ }^{2} S_{1 / 2}$ ground state, will be excited to higher electronic states. The decay from the lowest excited level $\left(2{ }^{2} P_{1 / 293 / 2}\right.$ doublet $)$ leads to photon emission at the Li resonance line of 670.8 $\mathrm{nm}$, which can be observed with excellent radial and temporal resolution. Since the ratio of the ionization function $\left\langle\sigma_{I} v_{e}\right\rangle$ and the excitation function $\left\langle\sigma_{s \rightarrow p} v_{e}\right\rangle$ is only weakly depending on the plasma temperature above $10 \mathrm{eV}^{19}$ lithium beams offer excellent possibilities to monitor the temporal evolution of boundary layer $n_{e}$ profiles in tokamak discharges. The spatial resolution is about $1 \mathrm{~mm}$.

The Al/Li oven described above has been installed at the TEXTOR tokamak. Figure 8 shows the experimental arrangement. In order to achieve the necessary lithium flux density of $2 \times 10^{14}$ atoms $/ \mathrm{cm}^{2} \mathrm{~s}$ for a sufficient $\mathrm{Li}$ emission signal, the exit hole is moved to the position of the radius of the liner wall, the oven itself being placed on a movable supporting system. The oven temperature is kept constant within $3^{\circ}$ at $560^{\circ} \mathrm{C}$. As shown in Sec. I, the FWHM of the beam is $18 \mathrm{~mm}$ at a distance of $150 \mathrm{~mm}$. An automatic shatter prevents lithium from entering the torus between shots, as the 


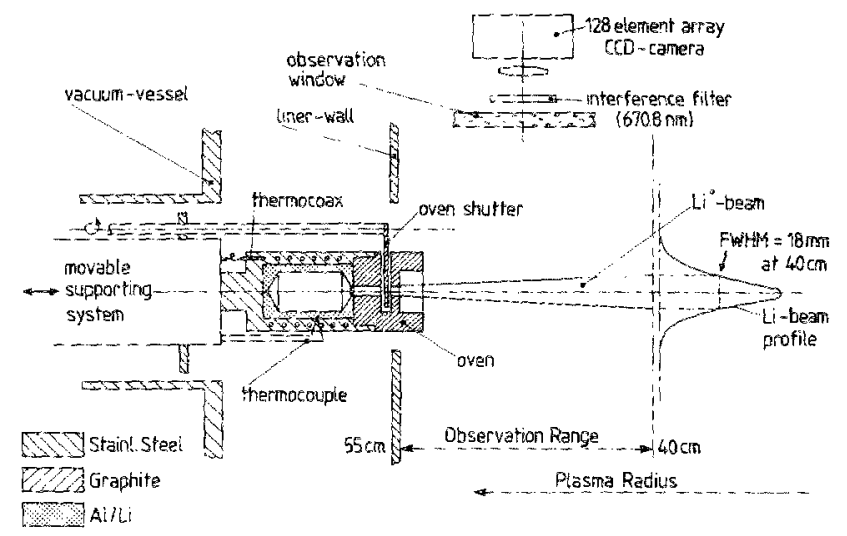

FIG. 8. Experimental setup of the Al/Li oven at TEXTOR: The oven is placed on a movable support unit, the effusion duct being positioned at the liner-wall radius. A shutter prevents Li from entering TEXTOR between discharges. The Li light emission profile is imaged onto a linear array $C C D$ camera.

contamination of the vacuum vessel with $L i$ should be minimized. Radial emission profiles of the Li resonance line are imaged onto a linear array CCD camera, consisting of 128 pixels (Reticon G128/10), the spatial resolution being 1.2 $\mathrm{mm}$ in radial and $12 \mathrm{~mm}$ in toroidal direction. These profiles can be recorded with a maximum repetition frequency of 2 $\mathrm{kHz}$.

Unwanted radiation, originating from atoms or molecules other than $L i$, has to be blocked by a narrow interference filter at $670.8 \mathrm{~nm}$. Background radiation from singly ionized oxygen atoms at $672.1 \mathrm{~nm}$ seems to be dominating. Therefore, the spectral width of the filter should not exceed 5 $\AA$. The oven surface surrounding the exit duct is exposed to the boundary plasma during shots. In order to reduce the oxygen density in the observation volume, the stainless-steel cap of the oven has been replaced with graphite. A typical electron density profle (TEXTOR shot No. 36000) measured with the setup described above is shown in Fig. 9. This discharge was heated only by the plasma current; no additional heating method was applied. The data curve was taken during the flat top phase at $t=1 \mathrm{~s}$, while the total shot duration was $3 \mathrm{~s}$. All limiters were positioned at a plasma radius of $46 \mathrm{~cm}$. The $n_{e}$ profile was derived from the emission profile by the algorhythm published in Ref 17 . Because the lithium-beam density decreases considerably in the observation volume (see data in Sec. I), the emission profile has to be convoluted with the flux decay curve of Fig. 6 . The radial beam profiles (Fig. 7) have also to be taken into consideration, as the toroidal extension of the camera array's image amounts to $12 \mathrm{~mm}$ in the observation plane. Up to now, we have achieved for a single $\mathrm{Al} / \mathrm{Li}$ insert more than 100 days of TEXTOR operation. Lithium fluxes decreased only very little during this time. The system is operated on an automatic basis, i.e., heating up in the morning and cooling down in the evening as well as starting the digital data-acquisition system is initiated by automatic devices.

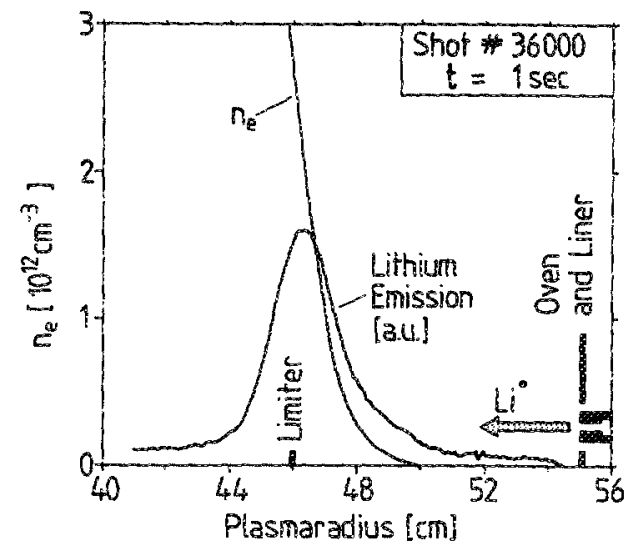

Fir. 9. A typical electron density profile in the scrape-oft layer of TEXTOR measured with the Al/Li oven (shot No. 36000). Here, all limiters were positioned at a plasma radius of $46 \mathrm{~cm}$. The profle was recorded $1 \mathrm{~s}$ after the start of the tokamak discharge in the fat top phase. The original light emission profile is also plotted.

In conclusion, we state that an easy to handle, compact thermal lithium-beam source has been developed and tested at TEXTOR, where electron density profiles have been obtained in the edge plasma region. Long operation periods of more than 100 days have been achieved, without the necessity of breaking the vacuum for maintenance and for refilling of $\mathrm{Al} / \mathrm{Li}$ material. As a future application, we intend to install at least one additional oven situated at a different poloidal position at TEXTOR.

'R. P. Schorn, H. L. Bay, E. Hintz, and B. Schweer, Appl. Phys. A 43, 147 (1987).

2A. J. McAlister, Bull. Alloy Phase Diagr. 3, 177 (1982).

${ }^{3}$ L. P. Costas, USAEC-Report TID-16676 (1963).

${ }^{4} \mathrm{C}$. J. Wen, Ph.D Dissertation, Stanford University, 1980

${ }^{5} \mathrm{G}$. Sauerbrey, Z. Phys. 355,206 (1959).

${ }^{6} \mathrm{E}$. Dulni, Mh.D. dissertation, University of Bochum, and KFA-Report Jül-1936, July 1984.

${ }^{7} \mathrm{~A} 1 / \mathrm{Li}$ 80/90F, Pechiney Corporation, Graf-Adolf-Strasse 41, D-4000 Düsseldorf, West Germany.

${ }^{8}$ F. Kohlrausch, Praktiche Fhysik (Teubner, Stuttgart, 1986), Vol.3, pp. 259-260.

"J. Roth and W. M. Wang (private communication).

1"S. Datz and E. H. Taykor, J. Chem. Phys. 25, 389 (1956).

"R. Bisenius, engineering diplona thesis, Fachhochschule Aachen, Abteilung Jülich, October $\$ 987$.

${ }^{12} \mathrm{~K}$. Kadota K. Matsunaga, H. Igushi, M. Fujwwara, K. Tsuchida, and $\mathbf{J}$ Fujita, Jpn. J. Appl. Phys. 21, L260 (1982).

1.3. Bogen, H. Hartwig, E. Hintz, K. Höthker, Y. T. Lie, A. Pospieszczyk, U. Summ, and W. Bieger, J. Nucl. Mater. 128 and 129, 157 (1984).

${ }^{14}$ Y. T. Lie, K. Hottker, W. Bieger, and K. Kadota, in Proceedings of the International Conference on Plasma Physics, Lausanne, Switzerland, 1984.

i5. Bogen and E. Hintz, in Physics of Plasmo- Wall Bnteractions in Controlled Fusion, edited by D. E. Post and R. Behrisch (Plenum. New York, 1986), p. $21 \%$

${ }^{16} \mathrm{~K}$. McCormick and the ASDEX-Team, Rev. Sci. Instrum. 56, 1063 (1985).

${ }^{17}$ A. Pospieszeryk and G. G. Ross, Rev. Sci. Instrum. 59, 605 (1988).

${ }^{14}$ A. Pospieszczyk, F. Aumayr, H. L. Bay, E. Hintz, P. Leismann, Y. T. Lie, G. G. Ross, D. Rusbüldt, R. P. Schorn, H. Schweer, and H. Winter, I. Nucl. Mater. 574, $162(1989)$.

19N. Brenning, 3. Quant. Spectrose. Radiat. Transfer 24, 298 ( 980 ). 